\title{
Design and Calculation of the Landing Track Preparation of Chang'e-3 Probe
}

\author{
Guangwei Yu ${ }^{1, a}$,Ting Zhang ${ }^{2, b}$,Daoming Feng ${ }^{3, c}$ \\ ${ }^{1 .}$ Wuhan Technology and Business University ,Wuhan ,Hubei, China \\ ${ }^{2}$ Wuhan Technology and Business University ,Wuhan ,Hubei , China \\ ${ }^{3}$ College of Mathematics and Computer Science Xinyu University Xinyu, China \\ asunnyygd@163.com, ${ }^{\mathrm{b}} 14523469 @ q q . c o m,{ }^{\mathrm{c}} 1647821068 @ q q . c o m$
}

Keywords:perilune; apolune; three dimensional coordinate system

\begin{abstract}
This paper discussed the problems in the soft landing of Chang'e-3 Probe on moon, established a Selenocentric celestial coordinate system, used the latitude and longitude coordinates coefficients conversion formula, calculated the location of the perilune and the apolune of Chang'e- 3 soft landing. The author divided the landing process into five stages: main decelerating phase, fast adjustment phase, crude avoidance phase, fine avoidance stage and free fall process. By using Newton movement theory and the conservation of energy, the author calculated the speed of Chang'e-3 at the perilune and the apolune, and then deduce the relationship of the above speed with the long radius and short radius of moon. The research in this paper makes preparation for further research into the implementation of landing.
\end{abstract}

\section{Description of the problem}

Chang'e-3 lunar probe is going to start its soft landing on the moon as planned on December 14, 2013. The way it lands and whether it can do it successfully has become a focus throughout the world.

On December 10, Beijing time, Chang'e-3 has successfully reduced into a planned lunar landing preparations orbit, and this is its last orbit adjustment. Prior to the implementation of the soft landing, Chang'e-3 will continue to fly stably on this orbit which is about 15 kilometers and 100 kilometers away from perilune and apolune respectively. During this stable flight, the landing sensors and landing data will be confirmed once again, and final preparations will be made for the starting height, speed, time of the soft landing.

The landing site of Chang'e-3 is in the relatively flat Rainbow Bay area. However, due to the uncertainty of the Lunar terrain, there will still exist some difficulties in the selection of the final landing place. It is reported that Chang'e-3 is going to fall parabolically from the place about 15 kilometers from the perilune, with its relative velocity gradually dropping from 1.7 kilometers per second to zero. The whole process will take more than 10 minutes.

Since there is no atmosphere on the surface of the moon, Chang'e-3 cannot rely on a parachute to land. As a result, it can only rely on variable thrust engine to perform such soft landing missions as mid-course corrections, brake, power reduction and hovering. It is reported that the main engine of Chang'e-3 lunar probe is currently China's biggest thrust engines, which can have adjustable thrust from 1500 to 7500 newton, and therefore could achieve the precision control of Chang'e-3.

In the space about 100 meters from the lunar surface, Chang'e-3 will perform brief hovering, scanning the lunar surface terrain, avoiding obstacles and finding landing sites. Then, Chang'e-3 will continue to fall 
gradually in the presence of anti-rocket, and hover again at the point about 4 meters from the lunar surface. At this time, it will turn off the recoil engine, and perform free fall. Because the probe owns landing buffer organization, and its several legs have elasticity, which ensures that it will not break while landing. After landing safely, Chang'e-3 will unfolder its solar panel to receive power, and the instruments carried will begin to work after testing and debugging. Subsequently, the "rabbit" lunar rover will leave the lander, conducting a 3-month scientific investigation on the lunar surface, while the lander will conduct detection where it lands.

\section{Problem analysis}

According to official data, Chang'e-3 is going to fall parabolically from the place about 15 kilometers from the perilune, with its relative velocity gradually dropping from 1.7 kilometers per second to zero. To determine landing preparation track in the perilune and in the apolune, inverse deduction can be done based on the longitude degrees of the landing point. According to a series of movements made by Chang'e-3 between the perilune and the lunar surface, the longitude of the perilune can be determined. Based on the Conservation of Mechanical Energy and the symmetry of parabola, the longitude of the apolune could be figured out. The direction of the tangent line of the perilune and the apolune is the direction of velocity of Chang'e-3 between this two points.

\section{3 model building and solution}

\section{A. The Position of Perilune and Apolune}

\section{(1)The establishment of Selenocentric celestial coordinate system}

When Chang'e-3 falls at perilune, it starts to reduce its power at the point about 15 kilometers from the perilune. So, taking the moon as the axis, the following Selenocentric celestial coordinate system [1] could be established.

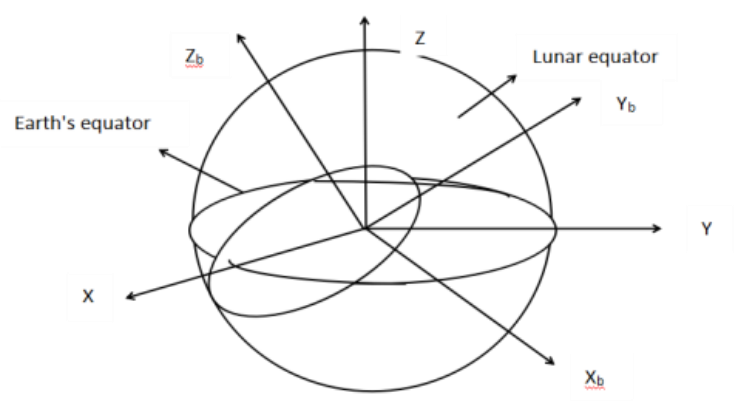

Fig. 1.Inertial coordinate system on the heart

In Figure 1, the origin is the lunar particle, parallel to the plane J2000 lunar equator, X-axis pointing J2000 flat equinox, $\mathrm{Z}$ axis refers to the North. 


\section{(2) The latitude and longitude coordinates of the conversion factor}

On the basis of Figure 1, we take latitude and longitude as the perilune point and apolune point respectively. After converting the latitude and longitude into coordinate coefficient, the following formula could be worked out ${ }^{[2]}$;

Let the moon long radius be a, short radius $\mathrm{b}$, flat rate countdown $\mathrm{c}$, then

$$
\begin{aligned}
& x=(d+h) \cos \alpha \cos \varphi \\
& y=(d+h) \cos \alpha \sin \varphi \\
& z=\left[\left(1-e^{2}\right) d+h\right] \sin \alpha
\end{aligned}
$$

In the above formulas: $\mathrm{d}$ is the prime vertical radius of curvature at latitude $\alpha, \quad d=a /\left(1-e^{2} \sin ^{2} \alpha\right)^{0.5}$

$\alpha$ and $\varphi$ are the latitude and longitude represented as coordinate points, $\mathrm{h}$ is the height relative to the lunar surface, e is the first eccentricity of the moon, $e^{2}=\left(a^{2}-b^{2}\right) / a^{2}=2 f-f^{2}$.

Note: $\mathrm{h}$ is the relative height of the moon, which is the height observed through the GPS satellite positioning, instead of the usual gravity associated with earth observation elevation values. Gravity-related height $(\mathrm{H})$ is usually relative sea level, or the height of a level surface. If gravity height $(\mathrm{H})$ is known, then while using the above formula, we should convert $\mathrm{H}$ into lunar height $\mathrm{h}=\mathrm{H}+\mathrm{N}$, where $\mathrm{N}$ is the relative height of the lunar surface for the geoid, $\mathrm{N}$ sometimes negative. The geoid is a surface similar to gravity at sea level.

\section{(3) Solving position at Perilune}

\section{(1) at position $3000 \mathrm{~m}$}

Landing point $p_{0}=\left(x_{0}, y_{0}\right)=(44.120 N, 19.510 W)$. A schematic view of the soft landing process is given in the official data. At 3000 meters from the lunar surface, the probe is almost above the target. Let P1 be the point at 3000 meters, then $p_{1}=\left(x_{1}, y_{1}\right)$, where ${ }^{p_{1}}$ 's longitude is the same as the longitude of landing point, so $y_{1}=y_{0}=19.510 \mathrm{~W}$.

Let the coordinates of Chang'e-3 at 15 kilometers away from perilune be $p_{2}=\left(x_{2}, y_{2}\right)$.

A circle around the moon on the lunar equator total 10913.9653 kilometers, while the circle is divided into $360^{\circ}$, and therefore the length of each degree at the equator is calculated as follows: $10913.9653 \mathrm{~km} / 360^{\circ}=30.3166 \mathrm{~km} / \mathrm{d}$

On the moon:

1)on the same longitude, a latitude difference means $30.32 \mathrm{~km}$; on the same latitude, a longitude difference means $30.32 \bullet \operatorname{cosp}$

2)For two points on the same latitude, their longitude distance difference is $s \bullet \cos \left(\theta_{0} \bullet \pi / 180\right)$

3) For two points on the same longitude, their latitude distance difference is 


$$
s \bullet \sin \left(\theta_{0} \bullet \pi / 180\right)
$$

4) degree offset in longitude: $\left[s \bullet \sin \left(\theta_{0} \bullet \pi / 180\right)\right] /\left[30.32 \bullet \cos \left(y_{1} \bullet \pi / 180\right)\right]$

5) degree offset in latitude: $\left[s \bullet \sin \left(\theta_{0} \bullet \pi / 180\right)\right] / 30.32$

6) obtained with the above formula:

$$
\begin{aligned}
& x_{2}=x_{1}+\left[s \bullet \sin \left(\theta_{0} \bullet \pi / 180\right)\right] /\left[30.32 \bullet \cos \left(y_{1} \bullet \pi / 180\right)\right] \\
& y_{2}=y_{1}+\left[s \bullet \cos \left(\theta_{0} \bullet \pi / 180\right)\right] / 30.32
\end{aligned}
$$

(8)

Wherein, $\mathrm{S}$ is the plane distance between two points, the unit: $\mathrm{km} ; \theta 0$ is the azimuth between two points, unit: degree, to the north as clockwise.

From (1), under the same longitude, ${ }^{p_{1}}$ latitude can be determined using the $p_{0}$ latitude, the expression formula is:

$$
\frac{1}{30.3}=\frac{\Delta x}{\Delta s}
$$

Based on $\Delta s=3$, we can figure out: $\Delta x=0.108$

Thereby: $x_{1}=x_{0}-0.108=44.012$

It follows that: $p_{1}=\left(x_{1}, y_{1}\right)=(44.012 N, 19.51 W) \quad$ (Coordinates at 3000 meters)

(2) the solution to ${ }^{x_{2}},{ }_{2}^{y_{2}}$ requires the calculation of the distance $\mathbf{S}$ between ${ }^{p_{1}}$ and ${ }^{p_{2}}$ :

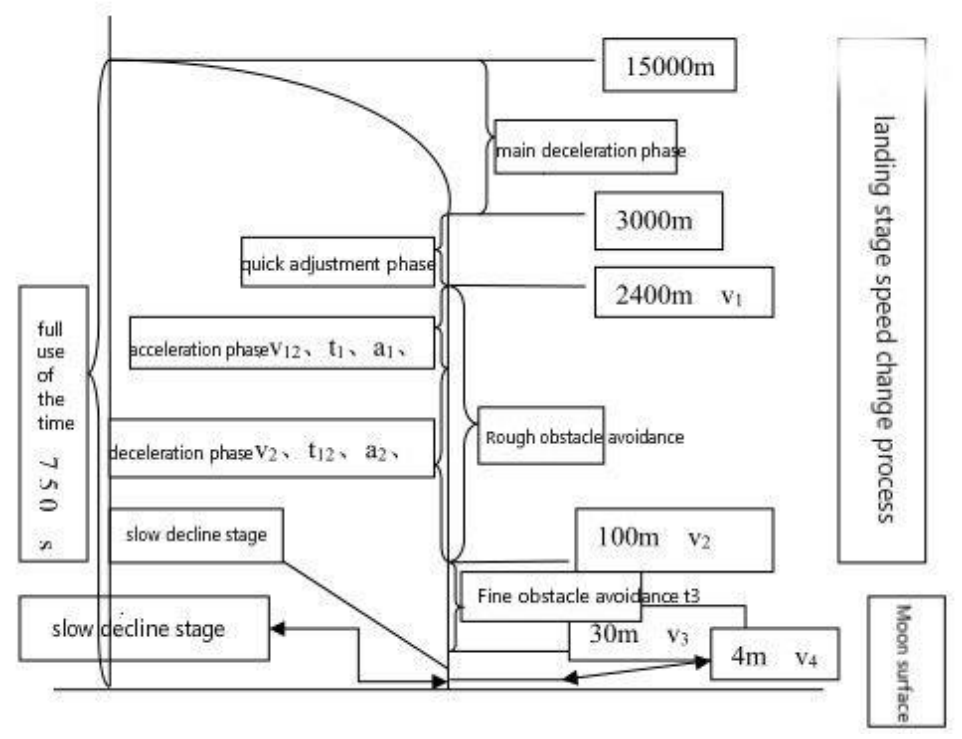

Fig. 2.speed change process

1)between $100 \mathrm{~m} \sim 2400 \mathrm{~m}$, namely crude avoidance phase, when the probe first accelerate and then 
decelerate. The main requirement is to avoid the big crater, to achieve hovering over $100 \mathrm{~m}$ at the landing site, and to initially identifu landing point:

$$
\left\{\begin{array}{l}
v_{12}=v_{1}+a_{1} t_{1} \\
h_{1}=v_{1} t_{1}+\frac{1}{2} a_{1} t_{1} \\
v_{2}=v_{12}+a_{2} t_{12} \\
h_{12}=v_{12} t_{12}+\frac{1}{2} a_{2} t_{12} \\
H=h_{1}+h_{12} \\
t_{2}=t_{1}+t_{12}
\end{array}\right.
$$

2)between $30 \mathrm{~m} \sim 100 \mathrm{~m}$, that is fine avoidance stage, when the probe decelerate uniformly. This stages requires Chang'e-3 to hover at a distance of $100 \mathrm{~m}$ above the lunar surface, capture images within $100 \mathrm{~m}$ range within the region near the landing point and get a three-dimensional digital elevation map. By analyzing the three-dimensional digital elevation map, it can avoid large craters, identify the best landing sites, and achieve zero horizontal velocity at $30 \mathrm{~m}$ above the landing site:

$$
\left\{\begin{array}{l}
v_{3}=v_{2}+a_{3} t_{3} \\
h_{2}=v_{2} t_{3}+\frac{1}{2} a_{3} t_{3}
\end{array}\right.
$$

3) $4 \mathrm{~m} \sim 30 \mathrm{~m}$ is the slowly declining stage, when the probe's velocity can be seen as uniform. The main task of this phase is to control the speed of the lander to be $0 \mathrm{~m} / \mathrm{s}$ at 4 meters above the lunar surface:

$$
h_{3}=v_{3} t_{4}
$$

4) $0 \sim 4 \mathrm{~m}$ is the free fall process, when the probe remains relatively stationary at 4 meters above the lunar surface, and then turns off the engine, so that Chang'e-3 free falls to precise point:

$$
\left\{\begin{array}{l}
v_{4}=v_{3}+g t_{5} \\
h_{4}=\frac{1}{2} g t_{5}^{2}
\end{array}\right.
$$

5)Through the above formula, the total time of the four stages can be obtained: $t_{2}+t_{3}+t_{4}+t_{5}=84.6 \mathrm{~s}$. The time for Chang'e-3 from 15 kilometers from perilune to the landing point: $t_{1}=t-\left(t_{2}+t_{3}+t_{4}+t_{5}\right)=665.4 \mathrm{~s}$, The main phase of slowing down (from P1 to P2) requires about 600 seconds.

6)Combined with equations (1) to (13), the distance $\mathrm{S}$ between P1 and P2 can be obtained through differential method:

$$
\left\{\begin{array}{l}
\int_{0}^{t} F d t=m v_{2}-m v_{1} \\
\frac{1}{2} m v_{2}^{2}-\frac{1}{2} m v_{1}^{2}=\int F d s+m g h
\end{array}\right.
$$

Where, $\mathrm{F}$ is the thrust force, $v_{1}=1.7 \mathrm{~km} / \mathrm{s}$ is the initial speed in perilune, $v_{2}=57 \mathrm{~km} / \mathrm{s}$ is the speed at $3000 \mathrm{~m}$ 
high. Therefore, $s=584.418 \mathrm{~km}$

Combining equation (8) to (14), we can obtain the latitude and longitude coordinates of perilune $p_{2}=\left(x_{2}, y_{2}\right)=(25.33 N, 19.864 W)$

\section{(4) Solving position at Apolune}

Apolune is in the same long axis of an ellipse as the perilune. Hence, let apolune coordinates be: $p_{3}=\left(x_{3}, y_{3}\right), y_{3}=y_{2}=19.864 \mathrm{~W}$

Moon's equatorial radius is $1737.646 \mathrm{~km}$, so the distance between perilune and apolune $s_{1}=1737.646 \bullet 2+115=3590.292 \mathrm{~km}$

Through formula $^{(2)}, x_{3}=53.633 S$

So, the latitude and longitude coordinates of Apolune is: $p_{3}=\left(x_{3}, y_{3}\right)=(53.633 S, 19.864 \mathrm{~W})$

\section{B. Speed of Chang'e-3 at the perilune and the apolune}

The movement of planets follows Kepler's three laws. This paper tries to explore the speed of Chang'e-3 at perilune and apolune from the perspective of energy. This method is called the Energy Method [5].

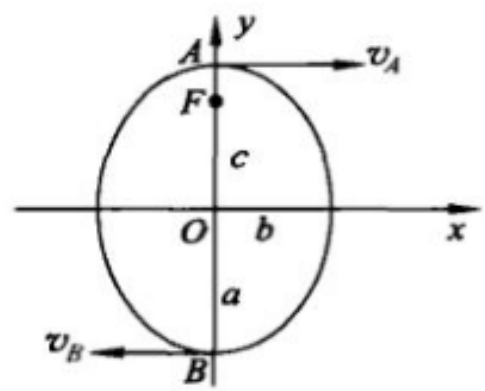

Fig. 3.Chang'e-3 in the moon's elliptical orbit

In figure 3, the focus of the ellipse $\mathrm{F}$ is the position of the moon, $v_{A}$ is the speed at the perilune, ${ }_{B}$ is the speed at the apolune. The figure shows the direction of $v_{A}$ and $v_{B}$ are perpendicular to the long axis of the moon, and the distance of point $\mathrm{A}$ and $\mathrm{B}$ to the sun are as follows:

$L_{A}=a-c, L_{B}=a+c$. At point $\mathrm{A}$ and $\mathrm{B}$, select an equal extremely short time $\mathrm{t}$, then the area surrounded by Chang'e-3 and the two line segments between Chang'e-3 and the moon are as follows:

$$
\begin{aligned}
& S_{A}=\frac{1}{2} \bullet v_{A} \bullet t \bullet L_{A} \\
& S_{B}=\frac{1}{2} \bullet v_{B} \bullet t \bullet L_{B}
\end{aligned}
$$

According to Kepler's second law: the area swept by the line segment planets and sun within equal time 
are the same, so $S_{A}=S_{B}$, this means:

$$
v_{B}=\frac{a-c}{a+c} \bullet v_{A}
$$

While moving, the total mechanical energy of Chang'e-3 equals the sum of kinetic energy and gravitational potential energy, so the mechanical energy of Chang'e- 3 while passing point $\mathrm{A}$ and $\mathrm{B}$ is:

$$
\begin{aligned}
& E_{A}=\frac{1}{2} m v_{A}^{2}+\left(-\frac{G M m}{L_{A}}\right)=\frac{1}{2} m v_{A}^{2}-\frac{G M m}{a-c} \\
& E_{B}=\frac{1}{2} m v_{B}^{2}+\left(-\frac{G M m}{L_{B}}\right)=\frac{1}{2} m v_{B}^{2}-\frac{G M m}{a+c}
\end{aligned}
$$

Because Chang'e-3 is subject to the gravitational force of the moon while orbiting the moon, based on mechanical energy conservation law, we can obtain:

$$
E_{A}=E_{B}
$$

Combining formula (17) to (19), we can obtain:

$$
\left\{\begin{array}{l}
v_{A}=\sqrt{\frac{(a+c) G M}{(a-c) a}} \\
v_{B}=\sqrt{\frac{(a-c) G M}{(a+c) a}}
\end{array}\right.
$$

After simplification:

$$
\left\{\begin{array}{l}
v_{A}=\frac{b}{a-c} \cdot \sqrt{\frac{G M}{a}} \\
v_{B}=\frac{b}{a+c} \cdot \sqrt{\frac{G M}{a}}
\end{array}\right.
$$

In the above speed formula, evidently $v_{A}>v_{B}$.

$b=a \bullet \cos \theta_{1}, c=a \bullet \sin \theta_{2}$, so $v_{A}=1.73 \mathrm{~km} / \mathrm{s}, v_{B}=1.68 \mathrm{~km} / \mathrm{s}$.

\section{Conclusions}

While calculating the speed at the perilune and the apolune, energy law, Kepler's second law can be used to find out the relationship between the speed at the perilune and the apolune. Finally problems could be solved based on the conservation of mechanical energy in a system.

\section{References}

[1] Yongping Ma, "lunar probe flight control", Beijing: National Defense Industry Press, 2010 
[2] Qingfen Dai translator, "coordinate transformation", Defense Petroleum Technology software development company, 2001

[3] Yafei Le, "based on GPS latitude and longitude of the space and the relative orientation of the distance calculation method," Chinese high-tech enterprises, 2012

[4] Kozai Y.The motion of a close earth satellite.Astron.J.1959,64(9): 367 377

[5] Jianwei Wang,Xing Li , "perihelion and aphelion speed of two typical method for finding", Physics Teacher, 2013

[6] Zhendong qu, Chang E III schematic orbit in recent months, 2013 